\title{
Ionized gas dynamics in the inner 2 pc of Sgr A West
}

\author{
John H. Lacy ${ }^{1}$, Wesley T. Irons ${ }^{2}$ and Matthew J. Richter ${ }^{3}$ \\ ${ }^{1}$ Department of Astronomy, University of Texas, Austin, TX, USA \\ email: lacy@astro.as .utexas.edu \\ ${ }^{2}$ Dept. of Physics and Astronomy, Johns Hopkins University, Baltimore, MD, USA \\ email: wirons@pha.jhu.edu \\ ${ }^{3}$ Department of Physics, University of California, Davis, CA, USA \\ email: richter@physics.ucdavis.edu
}

\begin{abstract}
We present a data cube of the [NeII] $(12.8 \mu \mathrm{m})$ emission from the inner $2 \mathrm{pc}$ of Sgr A West with $1^{\prime \prime}$ and $4 \mathrm{~km} \mathrm{~s}^{-1}$ resolution, and with substantially better SNR and velocity resolution than previous observations of the ionized gas. We compare the observations to two proposed models of the gas motions and distribution: flows along tidally stretched streamers, and more nearly circular motions with density wave compression. The density wave model provides a considerably better fit to the kinematics of the northern arm and western arc. Neither model fits the eastern arm and bar kinematics well.

To help understand the origin of the spiral pattern we calculated orbits in the potential of a black hole in a star cluster and find that the orbits naturally evolve to set up a onearmed spiral wave very similar to that observed, both spatially and kinematically. Magnetic or other perturbing forces may influence the formation of the spiral wave, but self gravity is not required. Because a density wave evolves on the orbit precession timescale, rather than the orbital timescale, a wave pattern should persist for several $10^{5} \mathrm{yr}$. No net inward motion of the gas is required by the model. If there is inflow, it is much smaller than is suggested by the infalling streamer model.
\end{abstract}

\section{Introduction}

Observations of the ionized gas motions in Sgr A West provided the first estimate of the mass of the black hole at the Galactic center (Lacy et al. 1980). The black hole mass is now much more precisely determined from stellar orbits, but the gas motions are still of interest for understanding how mass flows into a nuclear black hole.

Numerous authors have presented observations of the ionized gas, using infrared forbidden lines and infrared and radio recombination lines (Wollman et al. 1977, Lacy et al. 1980, Serabyn \& Lacy 1985, Lacy et al. 1991, Irons et al. 2012, Roberts \& Goss 1993, Paumard et al. 2004, Zhao et al. 2009, Zhao et al. 2010). The observations do not differ greatly, except in gradually improving $\mathrm{S} / \mathrm{N}$ and resolution, but the different authors have used different 3-d models to explain the observations. We will concentrate on two types of models: infalling tidally stretched streamers, with gas motions along the streamers, and a density wave, with gas motions close to circular.

\section{2. [NeII] observations and comparison to models}

Irons et al. (2012) presented $4 \mathrm{~km} \mathrm{~s}^{-1}$ spectral and $1^{\prime \prime}$ spatial resolution observations of [NeII] $(12.8 \mu \mathrm{m})$ emission from Sgr A West. Their data cube can be downloaded from the $A p J$ (Irons et al. 2013). The [NeII] line has less thermal broadening than hydrogen 


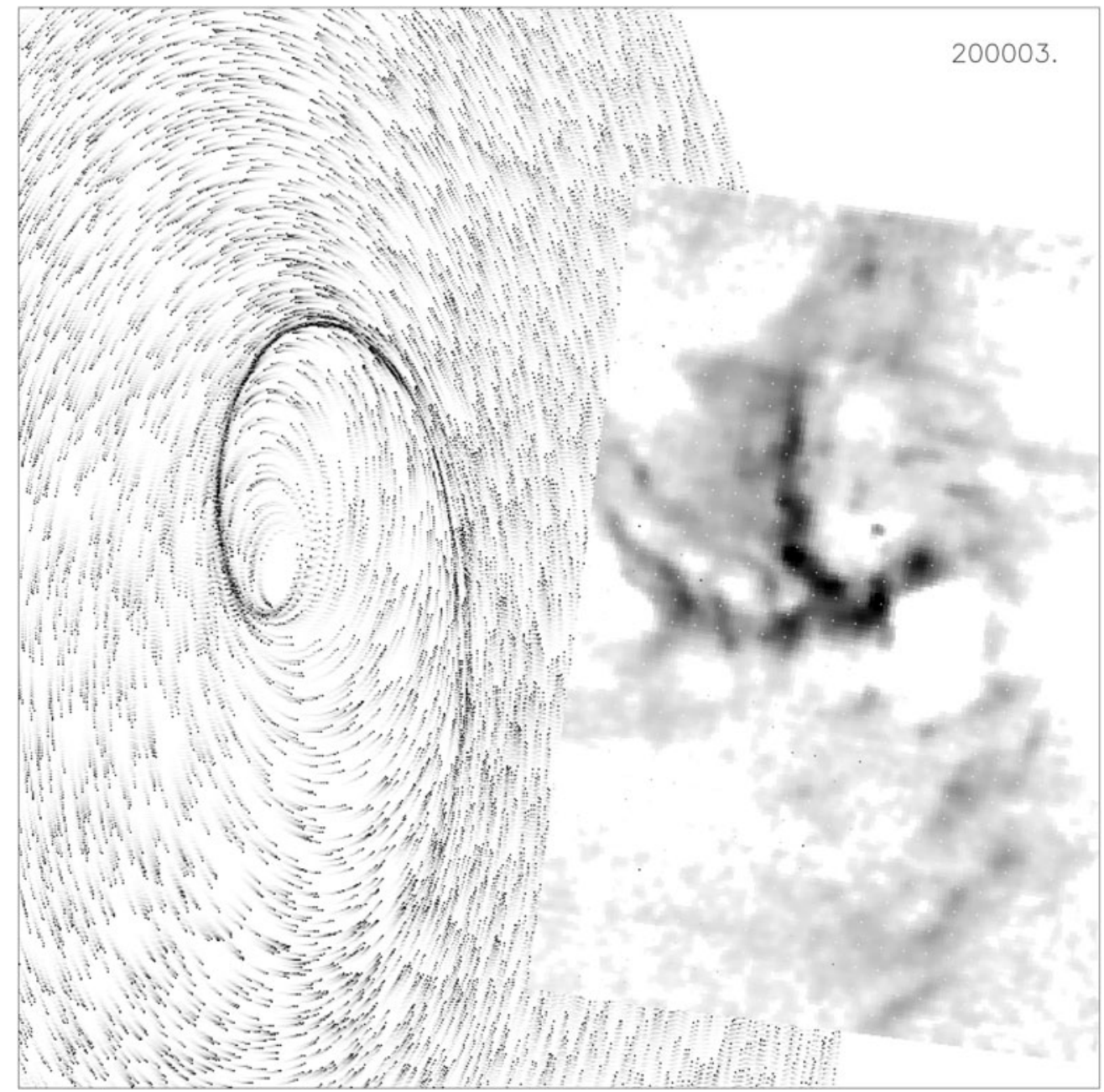

Figure 1. Distribution of particles in simulation after $2 \times 10^{5}$ yr and [NeII] emission. The [NeII] image is shown with a square root stretch. The simulation is tilted by a $65^{\circ}$ inclination angle.

recombination lines, but is otherwise very similar. Irons et al. (2012) compare their observations to two models: the model of Zhao et al. (2009) involving motions along the infalling legs of elliptical orbits, and a spiral wave model like that of Lacy et al. (1991) with nearly circular motions. They find that both models provide acceptable fits to the spatial distribution of the emission from the 'northern arm' and 'western arc', but that the spiral wave model provides a better fit to the kinematics of these features. The infalling streamer model fits the 'eastern arm' and 'bar' spatial distribution, whereas the spiral wave model does not, while neither model provides a very good fit to the kinematics of these features.

The discrepancy between the infalling streamer model and the observations can be seen most clearly in an angular position-velocity diagram (Irons et al. 2012). The predicted velocity pattern is offset in angle from the observed pattern in a way that indicates that the motions are more nearly circular than along the streamers. In addition, the best fit is obtained with a black hole mass less than that derived from stellar orbits. The spiral 


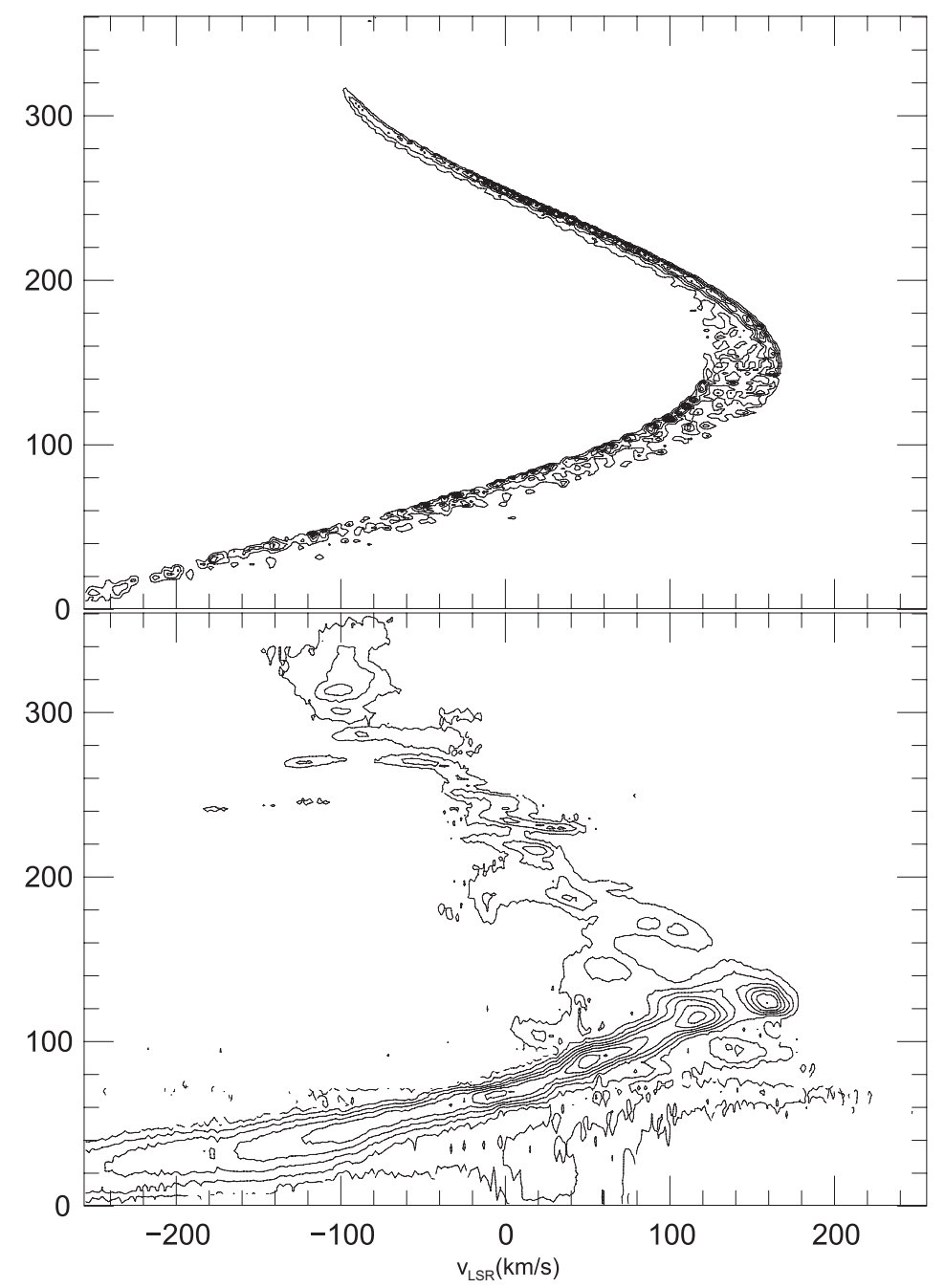

Figure 2. Position-velocity diagrams for the simulation and the [NeII] data. The vertical axis is angular position from Sgr $\mathrm{A}^{*}$ in the plane of the spiral or CND in degrees.

wave model provides the best fit if the gas orbits are close to circular, but tipped toward the center somewhat, and if the gas is moving at less than the circular orbital velocity.

\section{A physical explanation for the spiral}

The main problem with the spiral density wave model when it was first suggested by Lacy et al. (1991) was the lack of an explanation for how such a wave could be generated. A one-armed spiral is the dominant instability in a gravitational potential dominated by a point mass, but the mass of the gas in the inner 2 pc of Sgr A West is much too small to be gravitationally unstable. However, gravity may not be the perturbing force that organizes the gas into a spiral, and in fact, a perturbing force may not be required. To investigate how a spiral wave might form we carried out a simulation of particles orbiting in the potential of the $4.2 \times 10^{6} \mathrm{M}_{\odot}$ black hole and a cusp of stars. No interactions between the particles were included. Orbits in this potential are retrograde precessing 
ellipses. Whether the orbits close to the center precess faster or slower than orbits farther from the center depends on the steepness of the stellar cusp. The simulation started with the particles in aligned elliptical orbits, with all orbits having the same two foci, one on the black hole and the second offset by $0.1 \mathrm{pc}$. With a relatively flat stellar cusp (with a density power law shallower than $r^{-1}$ ) the outer orbits precessed more rapidly, causing orbit crowding along a trailing spiral, very similar to the northern arm and western arc. The distribution of particles after the simulation had run for $2 \times 10^{5}$ years is shown with the [NeII] image in Figure 1, and angular position-velocity diagrams of the simulation and the [NeII] data are shown in Figure 2. In the position-velocity diagrams, spectra were extracted from the simulated and observed data cubes along spirals following the northern arm and western arc emission ridges.

The simulated distribution corresponds rather closely both spatially and kinematically to the observations. It is particularly notable that the mass distribution used in the simulation is consistent with that derived from stellar motions. The black hole mass was constrained to agree with those observations, and the stellar distribution is within the allowed range. One prediction of the model is that the stellar cusp is rather flat. The facts that the gas velocities are somewhat smaller than circular orbital speeds in the potential of a $4.2 \times 10^{6} \mathrm{M}_{\odot}$ black hole and are tipped inward slightly from circular are explained by the fact that the compression caused by orbit crowding occurs just past apocenter.

The model shows that a spiral wave can develop without self gravity of the orbiting gas, and in fact without any other perturbing force. However, many orbital periods are required for precession to cause sufficient orbit crowding to cause a strong spiral. It may well be that forces other than gravity helped organize the gas into a spiral. In particular, magnetic forces may be important. There is a magnetic field of at least $1 \mathrm{mG}$ running along the northern arm (Aitken et al. 1998), which while not competing with gravity could help organize the gas orbits to form a spiral wave. Pressure forces must also be present, but they are probably even smaller than magnetic forces except perhaps in the northern arm where a shock may occur as the orbiting gas crosses the spiral wave. A more complete model of the gas motions in Sgr A West would require a magneto-hydrodynamic simulation.

Finally, it should be noted that the spiral wave model does not explain the distribution or kinematics of the gas in the eastern arm or bar regions. The gas there may be infalling or outflowing, and both gravitational and magnetic forces may affect its motion.

\section{References}

Aitken, D. K., Smith, C., Moore, T. J., \& Roche, P. F. 1998, MNRAS 299, 743

Irons, W. T., Lacy, J. H., \& Richter, M. J. 2012, ApJ 755, 90

Irons, W. T., Lacy, J. H., \& Richter, M. J. 2013, ApJ 771, 75

Lacy, J. H., Townes, C. H., Geballe, T. R., \& Hollenbach, D. J. 1980, ApJ 241, 132

Lacy, J. H., Achtermann, J. M., \& Serabyn, E. 1991, ApJ 380, L71

Paumard, T., Maillard, J. P., \& Morris, M. 2004, A\&A 426, 81

Roberts, D. A. \& Goss, W. M. 1993, ApJS 86, 133

Serabyn, E. \& Lacy, J. H. 1985, ApJ 293, 445

Wollman, E. R., Geballe, T. R., Lacy, J. H., Townes, C. H., \& Rank, D. M. 1977, ApJ 218, 103

Zhao, J-H., Morris, M. R., Goss, W. M., \& An, T. 2009, ApJ 699, 186

Zhao, Jun J-H., Blundell, R., Moran, J. M., Downes, D., Schuster, K. F., \& Marrone, D. P. 2010, ApJ 699, 186 